\title{
Modulation of x-ray line intensity emitted by a periodic structure under electron excitation
}

\author{
P. Jonnard, ${ }^{\text {a) }}$ J.-M. André, and C. Bonnelle \\ Laboratoire de Chimie Physique-Matière et Rayonnement, Université Pierre et Marie Curie, \\ UMR-CNRS 7614, 11 rue Pierre et Marie Curie, F-75231 Paris Cedex 05, France \\ F. Bridou and B. Pardo \\ Laboratoire Charles Fabry de l'Institut d'Optique, UMR-CNRS 8501, Batiment 503, \\ Centre Scientifique d'Orsay, BP 147, F-91403 Orsay Cedex, France
}

(Received 13 February 2002; accepted for publication 28 June 2002)

\begin{abstract}
We report on the intensity modulation of a characteristic x-ray line emitted by a periodic structure, as a function of the observation angle. This intensity variation takes place around the Bragg direction corresponding to the diffraction of the x-ray line by the emitting structure. An enhancement of the emitted radiation is observed and interpreted on the basis of the reciprocity theorem. The enhancement remains unchanged by varying the number of emitting periods. Following Yariv and Yeh [J. Opt. Soc. Am. 67, 438 (1977)], a possible application as x-ray resonator to achieve a distributed feedback soft $\mathrm{x}$-ray laser is envisaged. (C) 2002 American Institute of Physics. [DOI: 10.1063/1.1502189]
\end{abstract}

According to the dynamical theory of x-ray diffraction, a characteristic x-ray line emitted from an element inside a periodic structure can be diffracted by the same emitting structure under Bragg conditions. ${ }^{1}$ Interferences due to the diffraction process cause a modulation of the x-ray line intensity as a function of the exit angle in the narrow angular range satisfying the Bragg condition.

Variation as a function of the exit angle has already been observed and interpreted theoretically for the case of $\mathrm{x}$ rays emitted by a multilayer system irradiated by photons (fluorescence). This process was used to obtain information about the interfacial roughness. ${ }^{2}$ Analogy can also be made with the intensity variations of the $\mathrm{x}$-ray fluorescence observed under grazing exit condition. ${ }^{3,4}$ These variations had been interpreted in a coupled wave analysis by assuming that the field in the device consisted of two counter-running waves. ${ }^{5}$ These waves feed energy into each other due to the spatial modulation of the material. At the endpoints of the device a wave starts with zero amplitude receiving its initial energy through feedback from the other wave.

In order to study the dependence of the x-ray intensity emitted around the Bragg direction on the thickness of the emitter, we have analyzed the intensity of a characteristic line emitted by a periodic system as a function of the observation direction and of the energy of incident electrons. Indeed, because the electrons gradually lose their energy in the matter, it is possible to generate the $\mathrm{x}$ rays in the first periods or in all the periods of the multilayer.

We report here our results on the intensity variation of the $\mathrm{Si} K \alpha$ doublet emitted by a Mo/Si multilayer. The $\mathrm{Mo} / \mathrm{Si}$ multilayer is made of $40 \mathrm{Mo} / \mathrm{Si}$ bilayers deposited by electron beam evaporation on a Si substrate. The thicknesses of the Mo and Si layers are 1.6 and $3.2 \mathrm{~nm}$, respectively, leading to a superlattice period of $4.8 \mathrm{~nm}$.

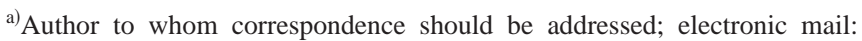
jonnard@ccr.jussieu.fr
}

An electron beam produced by a Pierce gun $(0-10 \mathrm{keV}$, $0-10 \mathrm{~mA}$ ) is used to ionize the Si $1 s$ core shell of the silicon atoms present in the sample. The irradiated surface is about 1 $\mathrm{cm}^{2}$. The experimental setup is such that the directions of the incident electrons and the detected photons are perpendicular. Then, the angle $\alpha$ between the sample surface and the direction of the detected photons is equal to the incidence angle of the electrons on the sample (Fig. 1). The $\mathrm{Mo} / \mathrm{Si}$ multilayer is glued with a silver paint on a rotary sample holder. Its rotation axis is perpendicular to the plane defined by the electron and photon directions (Fig. 1). This device enables us to vary the angle $\alpha$ around the Bragg angle of the $\mathrm{Mo} / \mathrm{Si}$ multilayer for the Si $K \alpha$ emission $(76.8 \mathrm{mrad})$ with a relative precision of $\pm 1.5 \mathrm{mrad}$. The Bragg angle is calculated by taking into account the refraction index correction. The Si $K \alpha$ emission $(2 p-1 s$ transition at $0.713 \mathrm{~nm})$ from the $\mathrm{Mo} / \mathrm{Si}$ multilayer is analyzed with a high-resolution Johann-type x-ray spectrometer ${ }^{6}$ using an $\mathrm{InSb}(111)$ crystal $(d=0.374 \mathrm{~nm})$ at the first reflection order. The acceptance angle by the spectrometer is $0.4 \mathrm{mrad}$.

The spectrometer is positioned at the peak of the $\mathrm{Si} K \alpha$

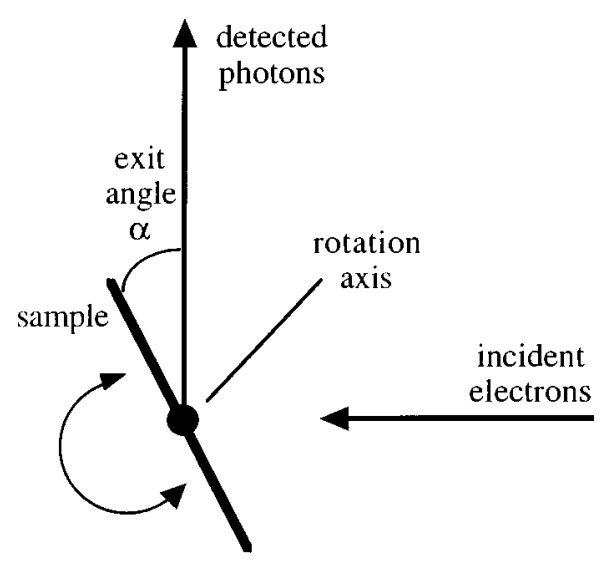

FIG. 1. Geometry of the experimental setup. 


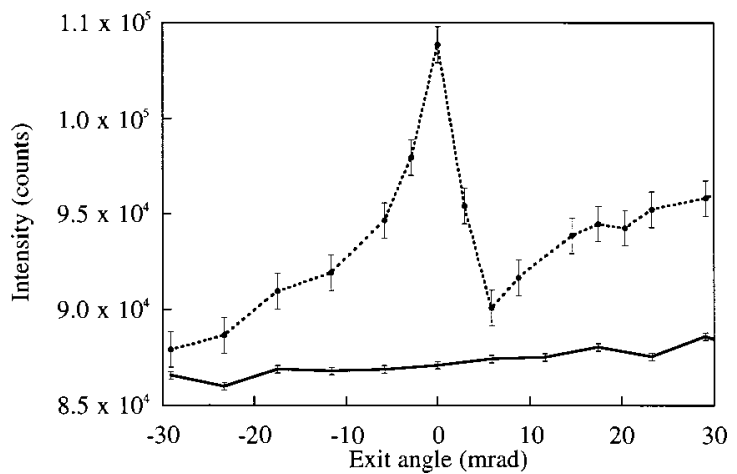

FIG. 2. Intensity of the Si $K \alpha$ emission measured as a function of the exit angle of the photons: for the $\mathrm{Mo} / \mathrm{Si}$ multilayer (dashed-dotted line) and for the Si crystal (solid line). The ordinates are the number of counts for an acquisition time of $10 \mathrm{~s}$ and an electron current density of $0.4 \mathrm{~mA} / \mathrm{cm}^{2}$.

emission and the intensity is measured as a function of the exit angle $\alpha$. This measurement is performed for $4 \mathrm{keV}$ incident electron energy, i.e., higher than the $\mathrm{Si} 1 s$ ionization threshold $(1.84 \mathrm{keV})$. A semiempirical model is used to estimate the depth distribution of the ionization for a bulk homogeneous material. ${ }^{7,8}$ The calculations are performed by simulating the multilayer structure with a 196-nm-thick $\mathrm{MoSi}_{2}$ film on a Si substrate. The ionizations are produced in the first $140 \mathrm{~nm}$, i.e., in the 30 first bilayers. Then, the silicone substrate is not involved in the x-ray emission spectrum.

In Fig. 2, we plot the distribution of the $\mathrm{Si} K \alpha$ radiation between 35 and $105 \mathrm{mrad}$, emitted by the multilayer at 4 $\mathrm{keV}$. It presents a maximum at an exit angle taken as zero in Fig. 2. This angle is near the Bragg angle of the $\mathrm{Mo} / \mathrm{Si}$ multilayer for the Si $K \alpha$ emission. A dip appears towards the larger angles of the maximum. A similar experiment performed at the same incident electron energy on a silicon single crystal only shows a monotonous behavior as a function of the exit angle. The observed intensity, normalized to take into account the number of emitting atoms, is plotted in Fig. 2. The relative intensity of the maximum with respect to the background has been estimated. This quantity, called the enhancement factor, is about $1.13 \pm 0.02$. The intensity modulation observed for the multilayer spreads over an angle range of about $30 \mathrm{mrad}$. By using the optical model presented in Ref. 9, we have estimated the angular width of the multilayer reflection curve to be of the same order. Then, the intensity modulation extends over the entire Bragg reflection range. One expects an increase of the enhancement factor with the narrowing of the multilayer reflection curve.

A calculated angular distribution is derived from a model similar to that previously implemented to describe the Bragg diffraction of the fluorescence emission in the multilayer. ${ }^{2}$ We consider that the excitation created by the incident electrons is independent of the depth, the slowing down of the electrons being neglected. To calculate the detected electromagnetic intensity $I_{d}$, the sample is supposed to be stratified, so that the concentration $C_{j}$ of the element $j$ depends only on the depth $z$. Then $I_{d}$ depends on $z$ and the direction $\theta_{d}$ of the emitted beam. Its value is determined by using the Helmoltz reciprocity theorem, ${ }^{10,11}$ which specifies that the electric field on the detector can be deduced linearly from the field $E_{d}$ produced at the point of the source by a fictitious dipole

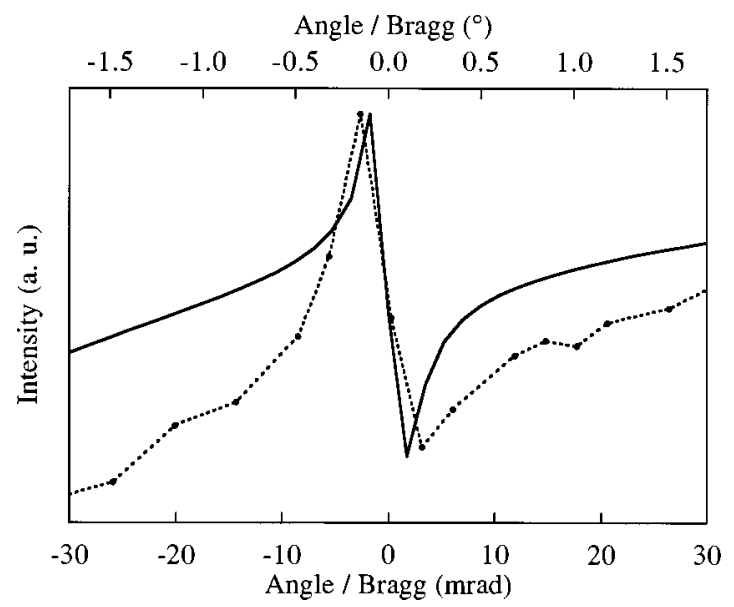

FIG. 3. Intensity of the $\mathrm{Si} K \alpha$ emission as a function of the exit angle for the Mo/Si multilayer: experiment (dashed-dotted line) and simulation (solid line).

situated at the position of the detector. The calculation is performed by using the standard matrix formalism suited for the optics of a multilayer medium. ${ }^{9,12}$ It results in the intensity emitted by the element $j$, measured in the direction $\theta_{d}$, being proportional to $C_{j}\left|E_{d}\left(\theta_{d}, z\right)\right|^{2}$.

The simulated intensity distribution is plotted in Fig. 3, and compared to the experimental one. In Fig. 3, the zero angle is the Bragg angle of the theoretical curve. The experimental curve is adjusted at the intensity jump of the theoretical curve, i.e., very close to the Bragg angle. The agreement between both curves is satisfactory in the modulation region. From the lower exit angles, the intensity increases up to a maximum for an $\alpha$ angle very near to the Bragg angle. Beyond, the outgoing intensity decreases rapidly and a dip is observed.

Observations for lower incident electron energy (2.1 $\mathrm{keV})$, i.e., for only four emitting bilayers, show an intensity modulation in the same angular range with the same enhancement factor. However, a change of the shape is observed, making the dip disappear. Such a modification of the shape would not be expected under x-ray excitation because no depth selectivity is observed with photons. From our results, a few periods are sufficient to provide the buildup of the enhancement. It can also be noticed that the maximum is located towards higher or lower angles according to whether the studied element is heavy or light.

An important point must be underlined: it concerns the possibility of using a periodic system simultaneously as emitter and resonator in the $\mathrm{x}$-ray range to achieve a distributed feedback laser, as suggested by Yariv ${ }^{13}$ and Yariv and Yeh. ${ }^{14} \mathrm{Up}$ to now, experiments developed with the aim of amplifying the soft $\mathrm{x}$-ray emissions have been performed by subjecting the $\mathrm{x}$-ray laser beam to one, or more, reflections on an independent optical system in the Fabry-Pérot optical scheme. ${ }^{15}$ Then, in this configuration the emitting and amplifying media were not the same.

To build a distributed feedback soft x-ray laser, the advantage of a multilayer-based device over the crystal-based proposed by Yariv, ${ }^{13}$ is that the superlattice period can be adapted to any wavelength in the soft $\mathrm{x}$-ray range. The possibility of implementing a multilayer structure as an efficient resonator is practically evidenced by our experiment. The 
next step consists in using as a lasing medium a multilayer structure, in which one of the two types of layer behaves as an amplifying medium with respect to the lasing wavelength.

${ }^{1}$ R. W. James, The Optical Principles of the Diffraction of X Rays (Bell, London, 1982), pp. 438-456.

${ }^{2}$ J.-P. Chauvineau and F. Bridou, J. Phys. IV 6, C7-53 (1996).

${ }^{3}$ R. S. Becker, J. A. Golovchenko, and J. R. Patel, Phys. Rev. Lett. 50, 153 (1983).

${ }^{4}$ T. Noma, A. Iida, and K. Sakurai, Phys. Rev. B 48, 17524 (1993).

${ }^{5}$ H. Kogelnik and C. V. Shank, J. Appl. Phys. 43, 2327 (1972).

${ }^{6}$ C. Bonnelle, F. Vergand, P. Jonnard, J.-M. André, P.-F. Staub, P. Avila, P.
Chargelègue, M.-F. Fontaine, D. Laporte, P. Paquier, A. Ringuenet, and B. Rodriguez, Rev. Sci. Instrum. 65, 3466 (1994).

${ }^{7}$ P.-F. Staub, X-Ray Spectrom. 27, 43 (1998).

${ }^{8}$ P.-F. Staub, P. Jonnard, F. Vergand, J. Thirion, and C. Bonnelle, X-Ray Spectrom. 27, 58 (1998).

${ }^{9}$ B. Pardo, T. Megademini, and J.-M. André, Rev. Phys. Appl. 23, 1579 (1988).

${ }^{10}$ H. A. Lorentz, Proc. Amsterdam Acad. 8, 401 (1905).

${ }^{11}$ P. Croce, L. Névot, and B. Pardo, C. R. Acad. Sci. Paris 274, 803 (1972).

${ }^{12}$ F. Abelès, Ann. Phys. 12, 706 (1950).

${ }^{13}$ A. Yariv, Appl. Phys. Lett. 25, 105 (1974).

${ }^{14}$ A. Yariv, and P. Yeh, J. Opt. Soc. Am. 67, 438 (1977).

${ }^{15}$ N. M. Ceglio, D. G. Stearns, D. P. Gaines, A. M. Hawryluk, and J. E. Trebes, Opt. Lett. 13, 108 (1988). 